\title{
IDENTIFICACIÓN Y CUANTIFICACIÓN DE HONGOS MICOTOXIGÉNICOS EN ALIMENTO PARA BOVINOS
}

\author{
René Santibáñez Escobar ${ }^{1}$, Margarita Hernández Gallardo ${ }^{2},{ }^{\circ}$ Oziel Dante Montañez Valdez ${ }^{1}$, José María Tapia González \\ José Alejandro Martínez Ibarra ${ }^{1}$, Juan Humberto Avellaneda Cevallos ${ }^{3,4}$, \\ ${ }^{1}$ Departamento de Desarrollo Regional, Centro Universitario del Sur, Universidad de Guadalajara, Av. Prolongación Colón \\ s/n Km 1 Ciudad Guzmán-Guadalajara Ciudad Guzmán Jalisco. ${ }^{\triangleright}$ montanez77@ hotmail.com \\ ${ }^{2}$ Departamento de Salud Pública, Centro Universitario de Ciencias Biológicas y Agropecuarias, Universidad de Guadalajara, \\ Km 15.5 carretera a Nogales, Predio Las Agujas, Zapopan Jalisco \\ ${ }^{3}$ Unidad de Investigación Cientifica y Tecnológica, Universidad Técnica Estatal de Quevedo, km 7 vía \\ Quevedo - El Empalme, C. P. 73. Mocache, Los Ríos, Ecuador \\ ${ }^{4}$ Escuela Superior Politécnica Agropecuaria de Manabi Manuel Félix López, Ecuador
}

\section{RESUMEN}

$\mathrm{S}^{\mathrm{e}}$ e identificó y cuantificó cepas fúngicas productoras de micotoxinas y grado de contaminación por aflatoxinas $\mathrm{B}_{1}, \mathrm{~B}_{2}, \mathrm{G}_{1}$ y $\mathrm{G}_{2}$, en alimento para bovinos en el municipio de Zapotlán, El Grande Jalisco, México. Se tomaron muestras de alimento para vacas lecheras y ganado de engorda de 30 unidades productoras. La identificación de las cepas fúngicas productoras de micotoxinas se realizó mediante caracterización macroscópica de colonias cultivadas bajo la técnica de vaciado en placa, y la identificación posterior de la morfología microscópica del hongo, para la cual se aislaron las cepas identificadas mediante la técnica de microcultivo. La cuantificación de cepas fúngicas se realizó mediante el conteo de unidades formadoras de colonias (UFC) y clasificadas bajo el criterio de tres escalas; a) Recuentos bajos $\left(10^{2}-10^{3}\right)$; b) Recuentos moderados $\left(10^{4}-10^{5}\right)$ y c) Recuentos altos $\left(10^{6}-10^{7}\right)$. Para la identificación del tipo de aflatoxinas se utilizó la técnica de cromatografía en capa fina. De las muestras procesadas se obtuvieron recuentos de UFC en la siguiente proporción: Recuentos altos $\left(10^{6}-10^{7}\right.$ UFC $\left.\mathrm{g}^{-1}\right) 56.66 \%$, recuentos moderados: $\left(10^{4}-10^{5} \mathrm{UFC} \mathrm{g}^{-1}\right)$ $36.66 \%$, recuentos bajos: $\left(10^{2}-10^{3} \mathrm{UFC} \mathrm{g}^{-1}\right) 6.66 \%$. Se aislaron un total de 148 cepas fúngicas, correspondiendo los porcentajes más altos a los siguientes géneros: Cladosporium spp., Penicillium spp. y Aspergillus spp. Se detectaron $33.33 \%$ de muestras positivas a aflatoxinas, dentro de las cuales se identificaron $B_{1}, B_{2}, G_{1}$ y $G_{2}$, todas en alta concentración, con $46.66 \%$ de muestras con fluorescencia positiva que no correspondieron a los estándares de aflatoxinas y $20 \%$ de muestras negativas.

Palabras claves: Bovinos, Alimento, Aflatoxinas

Recibido: 8-Junio-2011. Recibido en forma corregida: 23-junio-2011. Aceptado: 23-Junio-2011.

Publicado como ARTíCULO en Ciencia y Tecnología 4(1): 19-23. 2011
Abstract

Tentified and quantified Mycotoxin-producing fungal strains was identified and quantified, and the degree of contamination of aflatoxins $B_{1}, B_{2}, G_{1}$ and $G_{2}$, in the cattle food in the town of Zapotlán El Grande Jalisco, México was determined. Samples were fed to dairy cows and beef cattle 30 production units. The identification of mycotoxin-producing fungal strains was performed by characterizing macroscopic colonies grown under the plate casting technique, and the subsequent identification of the microscopic morphology of the fungus, for which isolated the strains identified by the technique microculture. Quantification of fungal strains was performed by counting colony forming units (CFU) and classified under the criteria of three scales: a) low counts $\left.\left(10^{2}-10^{3}\right), b\right)$ moderate counts $\left(10^{4}-10^{5}\right)$ and c) high counts $\left(10^{6}-10^{7}\right)$. To identify the type of aflatoxin used the technique of thin layer chromatography. Of the samples were obtained CFU counts in the following proportions: high counts $\left(10^{6}-10^{7} \mathrm{CFU} \mathrm{\textrm {g } ^ { - 1 } )} 56.66 \%\right.$, moderate counts $\left(10^{4}-10^{5} \mathrm{CFU} \mathrm{g}^{-1}\right) 36.66 \%$ and low counts $\left(10^{2}-10^{3} \mathrm{CFU} \mathrm{g^{-1 }}\right) 6.66 \%$. A total of 148 fungal strains were isolated, the highest percentages correspond to the following genera: Cladosporium spp., Penicillium spp. and Aspergillus spp. $33.33 \%$ were detected aflatoxin positive samples, among which were identified $\mathrm{B}_{1}, \mathrm{~B}_{2}$, $\mathrm{G}_{1}$ and $\mathrm{G}_{2}$, all in high concentrations, also was obtained $46.66 \%$ of samples with positive fluorescence did not correspond to the standards of aflatoxins and $20 \%$ of negative samples.

Key words: Bovine, Feedstuff, aflatoxins. 


\section{INTRODUCCIÓN}

$\mathrm{L}$ a contaminación por hongos del alimento para bovino, causa serios problemas, dado que los tóxicos que producen, disminuyen la calidad del alimento y al mismo tiempo, la respuesta animal causando con ello una baja producción. Dentro de las sustancias tóxicas, se encuentran las micotoxinas, definidas como un metabolito tóxico secundario producido por un moho, entre las que destacan las aflatoxinas, las cuales han sido objeto de amplias investigaciones en los últimos años, especialmente en relación con sus efectos. Durante muchos años el nombre de Aspergillus flavus ha sido considerado como el único productor de aflatoxinas, pero en realidad es un grupo de especies de hongos los que pueden producir estos metabolitos (Flores et. al., 2006). Las aflatoxinas pueden formarse, en productos alimenticios antes y después de la cosecha, se han encontrado cada vez con mayor frecuencia en alimentos que se guardan en condiciones de humedad y temperaturas favorables a su desarrollo y en condiciones inadecuadas de almacenamiento. Las aflatoxinas han sido caracterizadas en cuatro grupos de distinto compuesto químico interrelacionados a los que se les llamó B (blue) y G (green) y los subíndices 1 y 2 para cada molécula química relativa a su movilidad cromatográfica. Estos compuestos pueden separar o adicionar un radical alcohol, hidroxilo, oxidrilo o una doble ligadura lo que da diferentes propiedades a cada uno de los compuestos (Peña y Duran 1990; Montemayor, 1993; Reyes et. al., 2009).

En el ganado productor de leche al ingerir dosis diarias de 2,300 a 4,200 ppb de aflatoxina $\mathrm{B}_{1}$, por un período de tres semanas a un mes producen un deterioro marcado en la salud y a la vez una reducción en el consumo de alimento, por lo que la producción de leche se disminuye. Además se ha reportado que cuando la aflatoxina $B_{1}$, es ingerida por bovinos, ésta inhibe las bacterias ruminales, reduce la digestibilidad de la celulosa y baja la concentración de ácidos grasos volátiles y la relación acético: propiónico (Hussein y Brasel, 2001; Masoero et al., 2007). La aflatoxina $\mathrm{M}_{1}$, es un metabolito de la $B_{1}$ que se produce en el hígado de un animal o persona que ha consumido alimento contaminado. La $\mathrm{M}_{1}$ es muy importante porque se acumula en leche, tejidos y huevo aproximadamente $24 \mathrm{~h}$ después del consumo de alimento, eliminándose en leche en una proporción de $300 \mu \mathrm{g}$ de $\mathrm{B}_{1} \mathrm{~kg}^{-1}$ alimento a $1 \mu \mathrm{g}$ de $\mathrm{M}_{1} \mathrm{~L}^{-1}$ de leche (Fernández, et. al., 1997; Montaño et al., 2007), sus efectos tóxicos son similares a los de $\mathrm{B}_{1}$, sobresaliendo los mutagénicos, hepatotóxicos, inmunosupresivos $\mathrm{y}$ carcinogénicos, por lo que ocasionan un serio problema de salud pública. Desafortunadamente uno de los mayores grupos consumidores de leche como son los infantes y adolecentes, son también los más sensibles a los efectos de la $\mathrm{M}_{1}$. Por lo anterior el objetivo de este trabajo es determinar y cuantificar la cantidad de hongos micotoxigénicos presentes en el alimento usado en las explotaciones bovinas.

\section{Materiales y Métodos}

T a investigación se llevó a cabo en treinta unidades Le producción bovina en el municipio de Zapotlán, El Grande Jalisco, México, en las que se colectaron 30 muestras de dos $\mathrm{kg}$ de alimento comercial o alimento elaborado y almacenado en la granja. Las muestras fueron enviadas para su procesamiento al Laboratorio de Salud Publica de la Facultad de Medicina Veterinaria y Zootecnia de la Universidad de Guadalajara. El tiempo que transcurrió del muestreo al análisis para cuantificar e identificar los hongos así como las aflatoxinas no fue mayor a $36 \mathrm{~h}$, para las siguientes determinaciones:

\section{Identificación de hongos mediante la técnica de cultivo de vaciado en placa.}

Preparación del medio de cultivo estéril utilizando $30 \mathrm{~g}$ de agar papa dextrosa en $1 \mathrm{~L}$ de agua. A cada $100 \mathrm{~mL}$ del medio se adiciona $1 \mathrm{~mL}$ de solución rosa de bengala al $0.6 \%$ y $0.5 \mathrm{~mL}$ de una solución de antibiótico, en este caso ampicilina de $200 \mathrm{mg}$ del antibiótico en $10 \mathrm{~mL}$ de agua destilada estéril. a) Vaciado de las placas. Se preparan las placas de cultivo estériles adicionando $15 \mathrm{~mL}$ del medio de cultivo. b) Se prepara una solución estéril de peptona de caseína al $0.1 \%$ con un $\mathrm{pH}$ ajustado a 7 para hacer la dilución de las muestras de alimento recolectadas. c) Dilución de la muestra. Se diluyen $10 \mathrm{~g}$ de la muestra de alimento en $90 \mathrm{~mL}$ del agua peptonada ( $1 \mathrm{~g}$ de peptona, $8.5 \mathrm{~g}$ de cloruro de sodio en un litro de agua ajustada a un $\mathrm{pH} 7$ con cloruro de sodio $1 \mathrm{~N}$ ) y de ésta se hacen diluciones de $10^{-2}, 10^{-3}$ y $10^{-4}$ inoculando $1 \mathrm{~mL}$ de cada dilución en las placas de cultivo. d) Incubación. Se llevan a la estufa de incubación a una temperatura de $20^{\circ} \mathrm{C}$ por un periodo de 72 a 120 horas. e) Microcultivo. De las cepas aisladas del crecimiento fúngico se inoculan en microcultivos de agar papa dextrosa y se incuban por un periodo de 72 a 120 horas para la identificación microscópica del hongo. f) Identificación de las cepas fúngicas. Los criterios que se utilizaron para la identificación de hongos productores de aflatoxinas, se basó en la caracterización macroscópica de las colonias fúngicas y la preparación de frótis húmedos a partir de microcultivos con el fin de determinar la morfología microscópica tomando en cuenta: identificación de 
hifas, identificación de esporas sexuales y asexuales, identificación de estructuras estromáticas, nutrición y crecimiento.

\section{Cuantificación de cepas fúngicas.}

La norma utilizada como criterio para clasificar la concentración de hongos en la mayoría de los alimentos terminados es la siguiente: $10^{2}-10^{3}$ recuentos bajos, $10^{4}-10^{5}$ recuentos moderados, $10^{6}$ $10^{7}$ recuentos altos. Estas interpretaciones se utilizaron para determinar los factores de propagación de las toxinas en el alimento para bovino.

Determinación cualitativa y cuantitativa de aflatoxinas $B_{1}, B_{2}, G_{1} y_{G_{2}}$,

Se realizó por el método de cromatografía en capa fina. Para la identificación de las cuatro aflatoxinas se basó en la observación de fluorescencia azul-verde bajo la luz ultravioleta de onda corta $(260 \mathrm{~nm})$ y onda larga $(360 \mathrm{~nm})$ tomando como patrón el estándar de referencia de la AOAC (1997).

\section{Resultados y Discusión}

\section{Identificación de cepas fúngicas.}

El 100\% de las muestras analizadas mostró contaminación por hongos, de las cuales se aislaron un total de 148 cepas. Las cepas encontradas se pueden clasificar en hongos de campo y hongos de almacén, entre los hongos de campo encontramos: Cladosporium, Fusarium y Alternaria, que causan problemas a las plantas y que son transmitidos de un ciclo a otro por las semillas. La principal diferencia entre los hongos de campo y los hongos de almacén son los requerimientos de agua para crecer. Los hongos de campo requieren humedades relativas de 90 a $100 \%$, en cambio los de almacén pueden crecer en humedades relativas de 65 a $90 \%$, condiciones de humedad muy frecuentes en el almacenamiento de granos (Carrillo, 2003). El presente trabajo enfocó su atención en aquellos hongos que producen alteraciones en los alimentos, así como las aflatoxinas, por ser consideradas las micotoxinas de mayor importancia en la producción animal. De las cepas aisladas se identificaron un total de 11 géneros distintos (Cuadro 1), de los cuales el $73.63 \%$ correspondió a Cladosporium spp., Penicillum spp., Aspergillus spp. y Fusarium spp., considerados todos como productores de aflatoxinas y otros tipos diferentes de aflatoxinas.

El género Cladosporium es un hongo de campo, sin embargo se le ha encontrado en mazorcas de maíz que se almacenan con altos contenidos

\section{Cuadro 1. Géneros identificados y porcentaje en- contrado}

\begin{tabular}{lc}
\hline \multicolumn{1}{c}{ GENERO } & $\mathbf{\%}$ \\
\hline Cladosporium spp. & 25.67 \\
Penicillum spp. & 21.62 \\
Aspergillus spp. & 16.89 \\
Fusarium spp. & 9.45 \\
Absidia spp. & 6.08 \\
Mucor spp. & 4.72 \\
Tricoderma spp. & 3.37 \\
Epicocum spp. & 2.02 \\
Paecelomices spp & 2.01 \\
Alternaria spp & 1.90 \\
Phoma spp. & 0.67 \\
Otros & 5.50 \\
\hline
\end{tabular}

de humedad. Tiene la capacidad de crecer a bajas temperaturas, aún bajo $0^{\circ} \mathrm{C}$, su capacidad toxígena no ha sido claramente definida. El Fusarium, para su desarrollo requiere actividad de agua de más de 0.9 , además, tiene una gran variedad de especies de importancia fisiopatológica (Martins and Martins, 2000). Este es junto con Aspergillus y Penicillum, uno de los géneros más importantes en la producción de micotoxinas que afectan a los animales domésticos (Hussein and Brasel, 2001). Al género Absidia y Mucor, se les encuentra como habitantes del suelo, y en materia orgánica en descomposición. Frecuentemente se aísla de granos, alimentos balanceados y sus ingredientes, esto concuerda con resultados obtenidos en este estudio al encontrarlo como contaminante del alimento para bovinos. Dado que los alimentos para animales tienen los nutrientes para cubrir las necesidades de los hongos, los factores ambientales de humedad y temperatura, son los responsables de acelerar su crecimiento y reproducción.

\section{Recuentos de unidades formadores de colonias.}

El $100 \%$ de las muestras analizadas, mostró contaminación por hongos, correspondiendo el $56.66 \%$ de los recuentos altos de $10^{6}-10^{7} \mathrm{~g}^{-1}$ unidades formadoras de colonias (UFC) recuentos moderados de $10^{4}-10^{5}$ UFC $\mathrm{g}^{-1}$ en un $36.66 \%$, recuentos bajos de $10^{2}-10^{3}$ UFC $\mathrm{g}^{-1}$ en un $6.66 \%$; además de acuerdo al número de cepas identificadas, cada muestra está contaminada en promedio por cinco cepas diferentes de hongos. Estos datos son de suma importancia para el análisis de los resultados obtenidos en la determinación de aflatoxinas. 


\section{Determinación cualitativa y cuantitativa de aflatoxinas.}

Se detectaron $33.33 \%$ de muestras positivas a aflatoxinas, encontrando en su mayoría la $\mathrm{B}_{1}$ en un

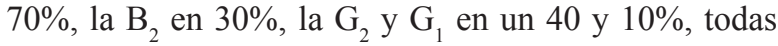
ellas con una concentración de 81 ppb. Además, se obtuvo un $46.66 \%$ de muestras que dieron lecturas con fluorescencia positiva que no correspondieron a los estándares de aflatoxinas y un $20 \%$ de muestras con lectura negativa. Aún cuando el $33.33 \%$ de las muestras se encontraron positivas a aflatoxinas hay que señalar que es un porcentaje elevado y más importante aún es que el $70 \%$ de las muestras positivas correspondieron a aflatoxina $\mathrm{B}_{1}$, que es metabolizada por los bovinos $\mathrm{y}$ eliminada con la leche en forma de aflatoxina $\mathrm{M}_{1}$, lo cual constituye un problema de salud pública ya que tiene efectos mutagénicos, hepatotóxicos, inmunosupresivos y carcinogénicos en quien la consume (Bazua, 2007), por lo que es recomendable establecer un sistema de control y vigilancia estableciendo un límite máximo de concentración de ésta aflatoxina en productos lácteos destinados para consumo humano como se ha establecido en otros países. De la misma forma, es recomendable establecer un sistema de control sobre los granos e ingredientes utilizados en la elaboración de alimento para animales y su almacenamiento ya que las concentraciones de aflatoxinas encontradas representan no solo un grave riesgo para la salud animal sino una considerable baja en la producción. Así mismo hay que considerar el potencial productor de aflatoxinas de las cepas encontradas como peligroso, ya que el $93.32 \%$ de las muestras mostraron alta y moderada contaminación por hongos. De igual forma se sugiere continuar las investigaciones en la identificación de otros tipos de micotoxinas considerando que un $46.66 \%$ de las muestras procesadas, mostraron fluorescencia positiva que no correspondió a los estándares de aflatoxinas y solo un $20 \%$ de las muestras fueron negativas. Actualmente se desarrollan nuevos métodos para la identificación y evaluación cuantitativa de las aflatoxinas en los tejidos animales tales como la cromatografía líquida de alta resolución (CLAR) para la determinación de aflatoxinas en alimentos para humanos y animales lo que ha representado un avance significativo sobre otros métodos de análisis, por su sensibilidad y precisión en la detección de diversos compuestos (Bermúdez et. al., 2002).

\section{Conclusión}

$\mathrm{C}$ on base en los resultados obtenidos podemos concluir que el total de las muestras analizadas mostró contaminación por hongos, con recuentos de unidades formadoras de colonias (UFC) de 56.6\%, encontrando principalmente el género Cladosporiun spp, seguido del Penicillum spp., Aspergillus spp. y Fusarium spp. encontrando concentraciones de las aflatoxinas sumamente elevadas, y totalmente fuera de los parámetros permitidos, por lo que es necesario la aplicación del reglamento para el control y vigilancia de la contaminación por hongos y aflatoxinas tanto en el alimento para consumo humano como animal.

\section{LiTERATURA CITADA}

AOAC, 1997. Official Methods of Analysis, 16th ed. Assoc. Offic. Anal. Chem. Arlington, VA, USA.

Bazua, R. 2007. Las micotoxinas, una amenaza constante en la alimentación animal. In: 1. Criterios de calidad de alimentos y su implicancia en la salud animal y humana. IX Encuentro de Nutrición y Producción en Animales Monogástricos, Montevideo, Uruguay. 21-28p.

Bermúdez, A. M., Espinosa P.A., Valenzuela Q. A. I. y Vázquez M.L. 2002. Extracción y determinación de aflatoxinas en muestras de hígado y músculo de cerdos Revista Científica, FCV-LUZ / 12(1) 5359.

Carrillo, L. Microbiología Agrícola. 2003. Universidad Nacional de Salta. (en línea). Consultado 30 May. 2011. Disponible en Mahttp:http://www. unsa.edu. ar/matbib

Fernández, A., Belio, R., Ramos, J. J, Sanz, M. C. and Saez, T. 1997. Aflatoxins and their metabolites in the tissues, faeces and urine from lambs feeding on an aflatoxin-contaminated diet. J. Sci. Food Agric. 74:161-168.

Flores, O. C. M., Hernández, P. L. B. y Vázquez M. J. 2006. Contaminación con micotoxinas en alimento balanceado y granos de uso pecuario en México en el año 2003. Tec Pecu. Méx 44:2247256.

Hussein, S. H. and Brasel, J. M. 2001. Toxicity, metabolism, and impact of mycotoxins on humans and animals. Review. Toxicology 167:101-134.

Martins, M. L. and Martins, H. M. 2000. Aflatoxin M1 in raw and ultrahigh temperature - treated milk commercialized. Food Addit Contam 17:871-874.

Masoero F, Galloa, A, Moschinia, M., Pivaa, G. and Diaza, D. 2007. Carryover of aflatoxin from feed to milk in dairy cows with low or high somatic cell counts. Animal 2007 1:1344-1350.

Montaño, P. B. V., Chirico, M. I. y Gemio R. 2007. Estudio toxicológico de presencia de aflatoxina M1 en leche bovina recolectada del municipio de Achacachi. Rev. Bol. Quim 24:90-94. 
Montemayor A. 1993. Micotoxinas, su importancia en salud humana y producción animal. Agrocultura 20: $20-21$.

Peña, D. S. y Durán, B. 1990. Efecto toxico de las aflatoxinas en la dieta. Ciencia y Desarrollo 16:6164.

Reyes, V. W., Martínez S.P, Espinoza, V. H. I., Nathalm V. M. A, De Lucas P. E. y Rojo, F. 2009. Aflatoxinas totales en raciones de bovinos y AFM1 en leche cruda obtenida en establos del estado de Jalisco, México. Tec Pecu. Méx. 47:223-230. 